\title{
Impact of Inter-modal Four-Wave Mixing on the Performance of Mode- and Wavelength-Division-Multiplexing Systems
}

\author{
Filipe Ferreira ${ }^{1}$, Daniel Fonseca ${ }^{2,3}$, Stylianos Sygletos ${ }^{1}$, Andrew Ellis ${ }^{1}$, Henrique Silva ${ }^{4}$ \\ ${ }^{1}$ Aston Institute of Photonic Technologies, Aston University, Birmingham, UK (f.ferreira@aston.ac.uk) \\ ${ }^{2}$ Coriant Portugal, Unipessoal Lda., 2720-093 Amadora, Portugal \\ ${ }^{3}$ Instituto de Telecomunicações, Technical University of Lisbon, 1049-001 Lisboa, Portugal \\ ${ }^{4}$ Instituto de Telecomunicações, DEEC, University of Coimbra, 3030-290 Coimbra, Portugal
}

\begin{abstract}
In this paper, we investigate the impact of inter-modal four-wave mixing on mode- and wavelength-divisionmultiplexing systems. A set of coupled nonlinear Schrödinger equations, including linear mode coupling, is derived allowing to isolate the inter-modal four-wave mixing terms. The efficiency of inter-modal four-wave mixing between degenerate LP modes is found to be significantly higher than the intra-modal four-wave mixing efficiency. However, it is shown that the inter-modal four-wave mixing efficiency between degenerate modes is significantly reduced by the linear mode coupling.
\end{abstract}

Keywords: Few-mode fibres, mode-division multiplexing, differential mode delay, four-wave mixing.

\section{INTRODUCTION}

Mode-division multiplexing (MDM) over few-mode fibres (FMFs) has been proposed as a next-generation solution to overcome the impeding installed capacity exhaustion of current single-mode fibres (SMFs) [1]. However, the guidance of multiple modes introduce impairments absent in SMFs, namely: differential mode delay (DMD) [2],[3], linear mode coupling (XT) [4] and inter-modal (IM) nonlinear (NL) effects [5]-[9]. The IM-NL effects are IM cross-phase modulation (IM-XPM) and IM four-wave mixing (IM-FWM).

Recent experimental investigations regarding propagation along FMFs have shown that IM-NLs are nonnegligible, namely IM-XPM and IM-FWM [5]. In [5], non-degenerated IM-FWM has been experimentally observed, demonstrating that IM-FWM can be fully matched over very large frequency separations with large chromatic dispersion in each mode contrarily to intra-modal FWM. Thereby, this paper is focused on the impact of IM-FWM in MDM-FMF systems.

The paper is organized as follows. In Section 2, a set of coupled nonlinear Schrödinger equations (CNLSE) is derived taking into consideration the existence of mode coupling between modes, extending the work that has been presented in [9]. Section 3 presents the assessment of the degradation for MDM-WDM transmission due to IMFWM. The main conclusions are gathered in Section 4.

\section{CNLSE DERIVATION AND ANALYSIS}

In order to find the nonlinear terms responsible for IM-NL between a specific set of channels, the total electric field has to be written as a sum over $M$ distinct modes, $N+1$ distinct frequencies, and 2 orthogonal polarizations. The total electric field vector in the frequency domain is given by:

$$
\tilde{\mathbf{E}}(\mathbf{r}, \omega)=\frac{1}{2} \tilde{E}_{\boldsymbol{x}}(\mathbf{r}, \omega) \hat{x}+\frac{1}{2} \tilde{E}_{y}(r, \omega) \hat{y}+c . c .=\sum_{d s l}\left[\frac{1}{2} \frac{F_{d}(x, y)}{\sqrt{N_{d}}} \tilde{A}_{d s l}\left(z, \omega-\omega_{s}\right) e^{-\mathrm{j} \beta_{d s l}^{(0)} z \hat{l}+c . c .}\right]
$$

where $d=\{1, \ldots, M\}$ is the mode index, $s=\{-N / 2, \ldots, N / 2\}$ is the frequency channel index ( $N$ even, without lack of generality), $l=\{1,2\}$ is the polarization index ( $l=1$ refers to $x$-polarization and $l=2$ refers to $y$ polarization), $\mathbf{r}$ is the position vector in Cartesian coordinates $(x, y, z), \omega$ is the angular frequency, $\hat{l}$ is the unit linear polarization vector, $\mathrm{j}$ is the imaginary unit, $\sum_{d s l}=\sum_{d=1}^{M} \sum_{s=-N / 2}^{N / 2} \sum_{l=1}^{2}, F_{d}(x, y)$ is the transversal field distribution of $d, \tilde{A}_{d s l}\left(z, \omega-\omega_{s}\right)$ is the Fourier transform of the slowly varying wave envelope $A_{d s l}(z, t)$ ( $t$ is the time variable), $N_{d}=\iint\left|F_{d}(x, y)\right|^{2} d x d y$ such that $F_{d}(x, y) / \sqrt{N_{d}}$ is the normalized electrical field distribution and $\left|A_{d s l}(z, t)\right|^{2}$ is the instantaneous optical power, $\beta_{d s l}^{(0)}$ is the value of the frequency dependent propagation constant $\beta_{d l}(\omega)$ at $\omega_{s}$, and $c$. c. stands for complex conjugate. Note that in $(1), F_{d}(x, y)$ is assumed to vary negligibly with $\omega$ within the C-band, small waveguide imperfections, polarization and nonlinear induced polarization [10]. $F_{d}(x, y)$ and $\beta_{d l}(\omega)$ are solutions of the eigenvalue equation [10].

The propagation of the total electric field in a FMF is governed by the wave equation [10]:

$$
\nabla^{2} \tilde{\mathbf{E}}(\mathbf{r}, \omega)=-\omega^{2} \mu_{0} \varepsilon_{p r}(x, y) \tilde{\mathbf{E}}(\mathbf{r}, \omega)-\omega^{2} \mu_{0} \widetilde{\mathbf{P}}_{N L}(\mathbf{r}, \omega)
$$

where $\widetilde{\mathbf{P}}_{N L}(\mathbf{r}, \omega)$ is the Fourier transform of the nonlinear polarization $\mathbf{P}_{N L}(\mathbf{r}, t)$ due to the third-order nonlinear silica susceptibility $\chi_{x x x x}^{(3)}$. Mode coupling is included in (2) considering waveguide imperfections along the fibre, $\varepsilon_{p r}(x, y)$ is replaced by $\varepsilon(x, y)=\varepsilon_{p r}(x, y)+\Delta \varepsilon(x, y)$ where $\Delta \varepsilon$ varies randomly in each fibre section [9]. 
The wave equation (2) can be solved replacing (1), the eigenvalue equation [10], the Taylor series of $\beta_{d l}(\omega)$ around $\omega_{s}$ and applying the inverse Fourier transform. Afterwards, considering the slowly varying envelope approximation (in space and time) [10], selecting mode $d$ by multiplying both sides by $F_{d}^{*}$ and integrating over the $x-y$ transverse plane, and selecting the terms oscillating at each specific frequency $\omega_{s}$ such that $\omega_{p}+\omega_{q}-\omega_{r}-$ $\omega_{s}=0$, a CNLSE can be written as:

$$
\begin{aligned}
& \left(\partial_{z}+\mathcal{D}+\frac{\alpha_{d s l}}{2}\right) A_{d s l}=-\mathrm{j} \sum_{a p i} \sum_{b q j} \sum_{c r k}\left[\begin{array}{c}
\xi_{i j k l} \gamma_{a b c d} A_{a p i} A_{b q j} A_{c r k}^{*} \\
\cdot e^{-\mathrm{j} \Delta \beta_{a p i, b q j, c r k, d s l} z}
\end{array}\right]-\mathrm{j} \sum_{a i} A_{a s i} C_{a s i, d s l} e^{\mathrm{j}\left(\beta_{d s l}^{(0)}-\beta_{a s i}^{(0)}\right) z} \\
& \mathcal{D}=\beta_{d s l}^{(1)} \partial_{t}-\mathrm{j} \beta_{d s l}^{(2)} / 2 \partial_{t}^{2}-\beta_{d s l}^{(3)} / 6 \partial_{t}^{3} \\
& \xi_{i j k l}=\delta_{i l} \delta_{j l} \delta_{k l}+2 / 3 \delta_{i l} \delta_{j l_{\perp}} \delta_{k l_{\perp}} \\
& +1 / 3 \delta_{i l_{\perp}} \delta_{j l_{\perp}} \delta_{k l} \\
& \gamma_{a b c d}=n_{2} \omega_{s} / c_{0} A_{a b c d}^{e f f} \\
& \beta_{d s l}^{(m)}=\left.\partial_{\omega}^{m} \beta_{d l}(\omega)\right|_{\omega=\omega_{s}}(m=1,2, \ldots) \\
& \Delta \beta_{a p i, b q j, c r k, d s l}=\beta_{a p i}^{(0)}+\beta_{b q j}^{(0)}-\beta_{c r k}^{(0)}-\beta_{d s l}^{(0)} \\
& C_{a s i, d s l}=\frac{\omega_{s}^{2} \mu_{0}}{2 \beta_{d s l}^{(0)}} \iint \Delta \varepsilon(x, y) \frac{F_{a} F_{d}^{*}}{\sqrt{N_{a} N_{d}}} d x d y
\end{aligned}
$$

where $\{a, b, c, d\}$ are mode indices, $\{p, q, r, s\}$ are frequency indices, $\{i, j, k, l\}$ are polarization indices, ${ }^{*}$ stands for complex conjugate; $\mathcal{D}$ in equation (4) is responsible for chromatic dispersion and DMD; $\beta_{d s l}^{(m)}$ in equation (5) is the value of the $m^{\text {th }}$ derivative of the frequency dependent propagation constant $\beta_{d l}(\omega)$ at $\omega_{s} ; \xi_{i j k l}$ in equation (6) groups the independent polarization combinations using the Kronecker delta function $\delta_{i l} ; \Delta \beta_{a p i, b q j, c r k, d s l}$ in equation (7) is the phase mismatch between waves api, bqj, crk and $d s l ; \gamma_{a b c d}$ in equation (8) is the nonlinear coefficient between modes ( $a, b, c, d) ; A_{a b c d}^{e f f}$ in equation (8) is the IM effective area $\left(A^{\text {eff }}\right)$ between modes $(a, b, c$, d) [10]; $n_{2}$ in equation (8) is the Kerr coefficient, approximately equal to $2.6 \cdot 10^{-20} \mathrm{~m}^{2} / \mathrm{W}$ [10]; $C_{a s i, d s l}$ in equation (9) is the linear mode coupling coefficient between mode $d$ and mode $a$. The first term on the right-hand side of (3) is responsible for all the NL effects taking place between the wave $d s l$ and the all the other $2 \times M \times(N+1)$ waves (including itself), this is, it includes all the terms responsible by: self-phase modulation (SPM), XPM, IMXPM, FWM, and IM-FWM. Note that SPM, XPM, and FWM refer to the intra-modal NL effects present in conventional SMF systems. The second term on right-hand side of (3) is responsible for the linear mode coupling.

The CNLSE (3) can be simplified considering the phase mismatch properties. Considering a common reference frequency $\omega_{0}$, the terms in the RHS of (7) can be written as $\beta_{a p i}^{(0)} \cong \beta_{a i}\left(\omega_{0}\right)-\beta_{a p i}^{(1)}\left(\omega_{0}-\omega_{p}\right)$, thereby:

$$
\Delta \beta_{a p i, b q j, c r k, d s l} \cong\left(\begin{array}{c}
\beta_{a i}\left(\omega_{0}\right)+\beta_{b j}\left(\omega_{0}\right) \\
-\beta_{c k}\left(\omega_{0}\right)-\beta_{d l}\left(\omega_{0}\right)
\end{array}\right)+\left(\begin{array}{c}
-\beta_{a p i}^{(1)} \cdot\left(\omega_{0}-\omega_{p}\right)-\beta_{b q j}^{(1)} \cdot\left(\omega_{0}-\omega_{q}\right) \\
+\beta_{c r k}^{(1)} \cdot\left(\omega_{0}-\omega_{k}\right)+\beta_{d s l}^{(1)} \cdot\left(\omega_{0}-\omega_{s}\right)
\end{array}\right)
$$

Assuming that $\left|\beta_{d l}\left(\omega_{0}\right)\right| \gg\left|\beta_{d s l}^{(1)} \cdot\left(\omega_{0}-\omega_{s}\right)\right|$ for any $d s l$ with $\omega_{s}$ and $\omega_{0}$ in the C-band, phase mismatch (10) can only be made approximately zero if the terms in the first parenthesis in the RHS of (10) can be made approximately zero. Furthermore, assuming that even for pairs of degenerate LP modes the effective index difference is not lower than $\sim 10^{-5}$ [4], [10], the possible mode matching conditions are $(a=m, b=n, c=n, d=m)$ and $(a=n, b=m$, $c=n, d=m$ ). Considering the abovementioned phase mismatch properties, the CNLSE are given by:

$$
\begin{aligned}
& \left(\partial_{z}+\mathcal{D}+\frac{\alpha_{m s l}}{2}\right) A_{m s l}=-\mathrm{j}\left(\begin{array}{c}
C_{S P M}+C_{X P M}+C_{F W M} \\
+C_{I M-X P M}+C_{I M-F W M}
\end{array}\right)-\mathrm{j} \sum_{n i} A_{n s i} C_{n s i, m s l} e^{\mathrm{j}\left(\beta_{m s l}^{(0)}-\beta_{n s i}^{(0)}\right) z} \\
& C_{S P M}=\gamma_{m m m m}\left|A_{m s l}\right|^{2} A_{m s l} \\
& =\sum_{p i} \sum_{q j} \sum_{r k \neq p i \wedge q j}\left(\begin{array}{c}
\xi_{i j k l} \gamma_{m m m m} A_{m p i} A_{m q j} \\
A_{m r k}^{*} e^{-\mathrm{j} \Delta \beta_{m p i, m q j, m r k, m s l} z}
\end{array}\right) \\
& =\sum_{n \neq m} \sum_{p i} \sum_{q j} \sum_{r k \neq q j}\left(\begin{array}{c}
\psi_{i j k l} \gamma_{m n n m} \\
\cdot A_{m p i} A_{n q j} A_{n r k}^{*} \\
\cdot e^{-\mathrm{j} \Delta \beta_{m p i, n q j, n r k, m s l^{z}}}
\end{array}\right) \\
& C_{X P M}=2 \gamma_{m m m m} \\
& \cdot\left(\sum_{p \neq s}\left|A_{m p l}\right|^{2}+\frac{1}{3} \sum_{p}\left|A_{m p l_{\perp}}\right|^{2}\right) A_{m s l} \\
& C_{I M-X P M}=2 \sum_{n \neq m} \sum_{p} \gamma_{m n n m}\left(\left|A_{n p l}\right|^{2}\right. \\
& \left.+\frac{1}{3}\left|A_{n p l_{\perp}}\right|^{2}\right) A_{m s l} \\
& \psi_{i j k l}=2 \delta_{i l} \delta_{j l} \delta_{k l}+\frac{2}{3} \delta_{i l} \delta_{j l_{\perp}} \delta_{k l_{\perp}}+\frac{2}{3} \delta_{i l_{\perp}} \delta_{j l} \delta_{k l_{\perp}} \\
& +\frac{2}{3} \delta_{i l_{\perp}} \delta_{j l_{\perp}} \delta_{k l}
\end{aligned}
$$

$C_{I M-F W M}$

In the first term on the RHS of (11) the nonlinear contributions due to SPM ( $\left.C_{S P M}\right)(12)$, XPM ( $\left.C_{X P M}\right)(13)$, FWM $\left(C_{F W M}\right)$ (14), IM-XPM ( $\left.C_{I M-X P M}\right)(15)$ and IM-FWM $\left(C_{I M-F W M}\right)(16)$ are identified. In order to solve (11), the splitstep Fourier method (SSFM) is modified for multimode propagation including linear mode coupling as in [9]. 
According to (14) and (16), the FWM-induced penalty is highly dependent on the phase mismatch. The smaller is the phase mismatch the larger is the induced penalty. The phase mismatch in (14) and (16) can be re-written using (7), a Taylor series around $\omega_{s}=\omega_{0}$ and the frequency condition for an idler in the central frequency $\left(\omega_{s}=\omega_{0}\right), r=$ $p+q$ (since $\omega_{p}=\omega_{0}+p \Delta \omega_{g}$ ), can be written as (ignoring the polarization dependency for convenience):

$$
\Delta \beta_{m p, n q, n r, m 0} / p \Delta \omega_{g} \cong-q \beta_{n 0}^{(2)} \Delta \omega_{g}-\left(\beta_{n 0}^{(1)}-\beta_{m 0}^{(1)}\right)-1 / 2 p \Delta \omega_{g}\left(\beta_{n 0}^{(2)}-\beta_{m 0}^{(2)}\right)
$$

Note that $p \neq 0$ in any case, since $p=r-q$ and $r \neq q$ ( $r$ and $q$ belong to mode $n$ ) see (14) and (16). On the RHS of (18): the first term is the walk-off induced by chromatic dispersion of mode $n$, the second term is the $D M D$ between modes $n$ and $m$, and the third term is proportional to the frequency slope of the $D M D$. In the intra-modal FWM case $(n=m)$, only the first RHS term of (18) is nonzero, and its minimum magnitude is $\beta_{n 0}^{(2)} \Delta \omega_{g}$ because $q$ cannot be zero according to (14) ( $q=r-p$ and $r \neq p$ since both belong to mode $m$ ). The IM-FWM case can be divided into three sub-cases:

(SC1) For pairs of degenerate modes (e.g., $L P_{11 a}$ and $\left.L P_{11 b}\right), \beta_{n 0}^{(1)} \cong \beta_{m 0}^{(1)}$ and $\beta_{n 0}^{(2)} \cong \beta_{m 0}^{(2)}$, the magnitude of the RHS of (18) is approximately $q \beta_{n 0}^{(2)} \Delta \omega_{g}$, similarly to FWM, but in this case $q$ can be zero $(r$ and $p$ belong to different modes). Therefore, the RHS of (18) is approximately zero for $q=0$ and any $p$ value between $-N / 2$ and $N / 2$ (except $p=0$ ). In such conditions, $N$ IM-FWM terms with negligible phase mismatch fall into the centre channel of $m$, inducing a high penalty in comparison to the other cases (independently of the DMD between the remaining modes that might be guided simultaneously).

(SC2) For pairs of non-degenerate modes (e.g., $\mathrm{LP}_{01}$ and $\mathrm{LP}_{11 \mathrm{a} / \mathrm{b}}$ ) with flat $\mathrm{DMD}, \beta_{n 0}^{(2)} \cong \beta_{m 0}^{(2)}$, only the third term in the RHS of (18) is zero. Therefore, the DMD values $\left(\beta_{n 0}^{(1)}-\beta_{m 0}^{(1)}\right)$ leading to maximum and minimum IM-FWM induced penalty are multiples of $\pm q \beta_{n 0}^{(2)} \Delta \omega_{g}$ and $\pm q \beta_{n 0}^{(2)} \Delta \omega_{g} / 2$.

(SC3) For pairs of non-degenerate modes (e.g., $\mathrm{LP}_{01}$ and $\mathrm{LP}_{11 \mathrm{a} / \mathrm{b}}$ ) with non-flat DMD, $\beta_{n 0}^{(2)} \neq \beta_{m 0}^{(2)}$, the third term in the RHS of (18) cannot be neglected, thereby the DMD values leading to maximum and minimum IM-FWM induced penalty identified for the flat DMD case are shifted.

\section{FWM/IM-FWM Impairments in MDM-WDM Transmission}

In this section, the performance of a MDM system is evaluated through the transmission of a WDM signal with $N+1$ carriers in each FMF mode. The figure of merit measured is the maximum input power (MP) such that the central channel of a given mode has a Q-factor of 7, assuming equal power on all carriers. The WDM carriers are intensity modulated at $10 \mathrm{~Gb} / \mathrm{s}$ by de Bruijn sequences with $2^{9}$ bits using a raised cosine pulse shape with a 0.5 roll-off. A WDM fixed grid cantered at $\omega_{0}$ and an angular frequency separation of $\Delta \omega_{g}$ is considered. Thereby, the frequency of a given carrier $s$, can be written as $\omega_{0}+s \Delta \omega_{g}$, with $s=\{-N / 2, \cdots, N / 2\}$.

The simulation setup considers a fibre length of $80 \mathrm{~km}$, a SSFM step of $50 \mathrm{~m}, N=80$ and a $50 \mathrm{GHz}$ spacing frequency grid. The FMF considered guides $3 \mathrm{LP}$ modes $\left(\mathrm{LP}_{01}, \mathrm{LP}_{11 \mathrm{a}}\right.$ and $\left.\mathrm{LP}_{11 \mathrm{~b}}\right)$, with zero DMD between $L \mathrm{P}_{11 \mathrm{a}}$ and $\mathrm{LP}_{11 \mathrm{~b}}$, and with a mode coupling strength such that the leakage from $\mathrm{LP}_{01}$ to the other modes at the end of $33 \mathrm{~km}$ is equal to $-18 \mathrm{~dB}$, matching the measurements in [11]. The remaining FMF characteristics at $1545 \mathrm{~nm}$ are: chromatic dispersion equal to $20 \mathrm{ps} /(\mathrm{nm} \cdot \mathrm{km})$ with a slope equal to $92 \mathrm{fs} /\left(\mathrm{nm}^{2} \cdot \mathrm{km}\right)$ for all modes, fibre loss equal to $0.22 \mathrm{~dB} / \mathrm{km}$ for all modes, and $A_{m n n m}^{\text {eff }}$ for pairs $(n, m)$ equal to $60 \mu \mathrm{m}^{2}$ for $\left(\mathrm{LP}_{01}, \mathrm{LP}_{01}\right), 80 \mu \mathrm{m}^{2}$ for $\left(\mathrm{LP}_{11 \mathrm{a}}, \mathrm{LP}_{11 \mathrm{a}}\right), 240 \mu \mathrm{m}^{2}$ for $\left(\mathrm{LP}_{11 \mathrm{~b}}, \mathrm{LP}_{11 \mathrm{a}}\right)$, and $120 \mu \mathrm{m}^{2}$ for $\left(\mathrm{LP}_{01}, \mathrm{LP}_{11 \mathrm{a}}\right)$.

In the following, the penalties induced by FWM and IM-FWM are compared. The MP is calculated solving (11) taking into account all IM-FWM terms involving modes $n$ and $m$ falling on the central frequency $s=0$ of mode $m$, with $n, m=\left\{\mathrm{LP}_{01}, \mathrm{LP}_{11 \mathrm{a}}, \mathrm{LP}_{11 \mathrm{~b}}\right\}$. Since the penalty induced on the central channels of modes $\mathrm{LP}_{11 \mathrm{a}}$ and $\mathrm{LP}_{11 \mathrm{~b}}$ are in average approximately equal, only the penalty for $\mathrm{LP}_{11 \mathrm{a}}$ is shown.

Fig. 1 shows $M P$ as a function of $D M D$ for each mode combination $(n, m), M P_{n, m}$. These results were obtained numerically solving (11) in the presence and absence of mode coupling. The results without mode coupling are analysed first. Fig. 1 (a) for $m=\mathrm{LP}_{11 \mathrm{a}}$ shows that IM-FWM with $n=\mathrm{LP}_{11 \mathrm{~b}}$ is the dominant penalty (lower $M P$ value), and that intra-modal FWM $\left(n=\mathrm{LP}_{11 \mathrm{a}}\right)$ is in fact leading to the lower penalty. The severe degradation due to IM-FWM is related with the high number of frequency combinations verifying the phase matching condition between degenerate modes, as explained above (SC1). Moreover, in Fig. 1 (a) can be seen that the IM-FWM induced penalty with $n=\mathrm{LP}_{01}$ (this is, IM-FWM between the pair of non-degenerate modes) is characterized by periodical notches at $D M D$ multiple of $\pm q \beta_{n 0}^{(2)} \Delta \omega_{g}$ as explained above (SC2). In practical cases, these notches may be reduced due to the variation of $D M D$ along the fibre length. Fig. 1 (b) shows that IM-FWM is the dominant source of penalty characterized by periodical notches at $D M D$ multiple of $\pm q \beta_{n 0}^{(2)} \Delta \omega_{g}$ (in practical cases these notches may be reduced as previously explained).

In the presence of mode coupling, Fig. 1 (b) shows that FWM and IM-FWM induced penalties in LP $\mathrm{P}_{01}$ are not affected significantly. However, Fig. 1 (a) shows a significant reduction of the penalty induced by IM-FWM 


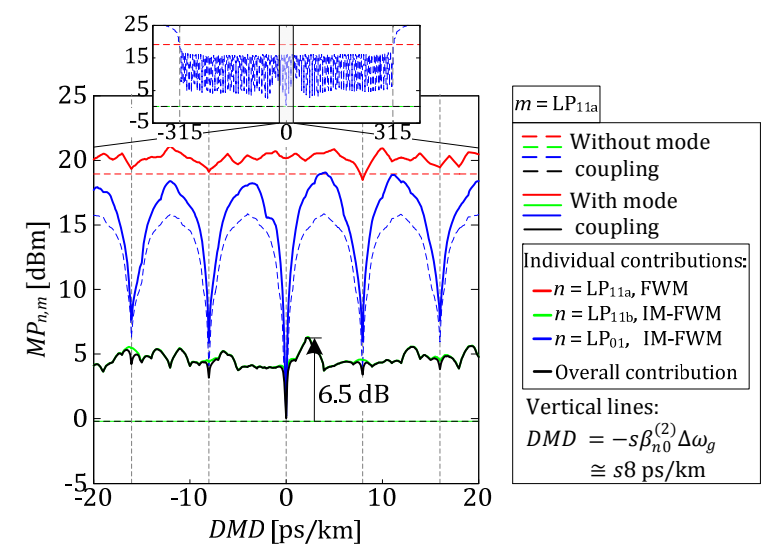

(a)

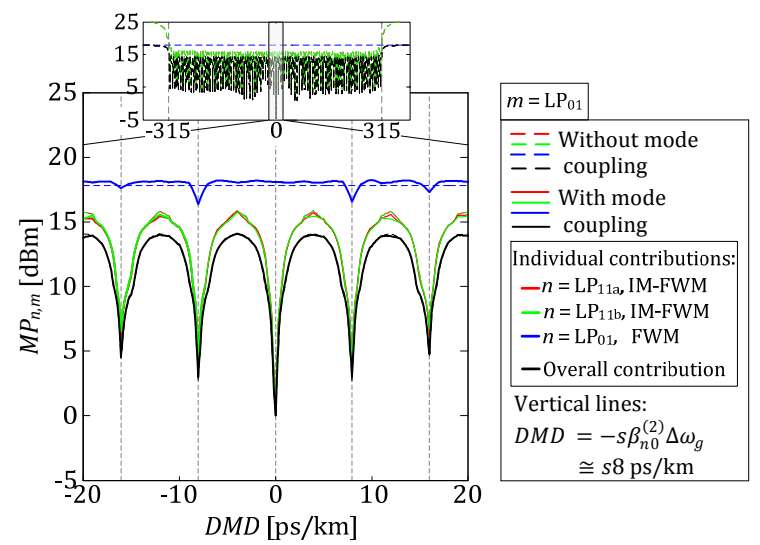

(b)

Figure 1. $M P_{n, m}$ as a function of DMD, for each pair of modes (n, $\left.m\right)$ : (a) $m=L P_{11 a}$ and (b) $m=L P_{01}$.

between $\mathrm{LP}_{11 \mathrm{a}}$ and $\mathrm{LP}_{11 \mathrm{~b}}$, the overall $\mathrm{MP}$ increase can reach $6.5 \mathrm{~dB}$. The different impact of mode coupling on the performance of the $\mathrm{LP}_{01}$ mode and of the $\mathrm{LP}_{11 \mathrm{a} / \mathrm{b}}$ modes is explained noting that, the mode coupling between $\mathrm{LP}_{11 \mathrm{a}}$ and $\mathrm{LP}_{11 \mathrm{~b}}$ is much stronger than between $\mathrm{LP}_{11 \mathrm{a} / \mathrm{b}}$ and $\mathrm{LP}_{01}$ [11], after some km's LP $\mathrm{P}_{11 \mathrm{a}}$ and $L \mathrm{P}_{11 \mathrm{~b}}$ are fully mixed.

Finally, the insets in Fig. 1 show that the IM-FWM results remain representative when increasing $D M D$ up to $\pm(N / 2) q \beta_{n 0}^{(2)} \Delta \omega_{g}(\sim 315 \mathrm{ps} / \mathrm{km})$, set by the frequency separation between the central channels and any of the extreme WDM channels $(s=0$ and $s= \pm N / 2)$.

\section{CONCLUSIONS}

In this paper, we investigated the impact of IM-FWM on MDM-WDM systems. For such purpose, a set of CNLSE including linear mode coupling was derived allowing to isolate the intra-modal FWM and IM-FWM terms. The efficiency of IM-FWM between degenerate LP modes is found to be significantly higher than the intra-modal FWM efficiency. However, it is shown that the IM-FWM efficiency between pairs of degenerate modes is significantly reduced by the linear mode coupling when considering mode coupling strengths presented in the literature. Finally, it has been concluded that, in order to have reduced IM-FWM efficiency between nondegenerate modes over the entire WDM grid, a DMD higher than $630 \mathrm{ps} / \mathrm{km}$ is required for 81 channels spaced of $50 \mathrm{GHz}$, considering a chromatic dispersion of $20 \mathrm{ps} /(\mathrm{km} \cdot \mathrm{nm})$, imposing difficulties for long-haul applications.

\section{ACKNOWLEDGEMENTS}

This work was supported in part by Coriant Portugal, Fundação para a Ciência e Tecnologia under grant SFRH/BDE/51094/2010, European Communities 7th Framework Programme under grant agreement 228033 (ModeGap) and grant agreement 619732 (INSPACE), and EPSRC under grant agreement EP/L000091/1 (PEACE).

\section{REFERENCES}

[1] R. Essiambre, and R. Tkach, “Capacity Trends and Limits of Optical Communication Networks,” Proceedings of the IEEE, vol. 100, no. 5, pp.1035-1055, May 2012.

[2] L. Gruner-Nielsen, et al., "Few Mode Transmission Fiber with low DGD, low Mode Coupling and low Loss," in Proc. OFC'2012, USA, paper PDP5A.1.

[3] F. Ferreira, D. Fonseca, H. Silva, "Design of Few-Mode Fibers With M-modes and Low Differential Mode Delay,” J. Lightwave Technol., vol.32, no.3, pp.353-360, 2014

[4] K. Ho, and J. Kahn, "Statistics of group delays in multimode fiber with strong mode coupling," J. Lightwave Technol., vol. 29, no. 21, pp. 3119-3128, 2011.

[5] R. Essiambre, et al., "Experimental Investigation of Inter-Modal Four-Wave Mixing in Few-Mode Fibers," IEEE Photon. Technol. Lett., vol. 25, no. 6, pp. 539-542, 2013.

[6] S. Mumtaz, R. Essiambre, and G. Agrawal, "Nonlinear propagation in multimode and multicore fibers: generalization of the Manakov equations,” J. Lightwave Technol., vol. 31, no. 3, pp. 398 - 406, 2013.

[7] N. Mac Suibhne, R. Watts, S. Sygletos, F. Gunning, L. Grüner-Nielsen, and A. D. Ellis, "Nonlinear Pulse Distortion in Few-Mode Fiber," in Proc. ECOC'2012, p. Th.2.F.5.

[8] A. Ellis, N. Mac Suibhne, F. Garcia Gunning, and S. Sygletos, "Expressions for the nonlinear transmission performance of multi-mode optical fiber," Opt. Express vol. 21, no. 19, pp. 22834-22846, 2013.

[9] F. Ferreira, S. Jansen, P. Monteiro, and H. Silva, "Nonlinear Semi-Analytical Model for Simulation of FewMode Fiber Transmission,” IEEE Photon. Technol. Lett., vol. 24, no. 4, pp. 240-242, 2012.

[10] G. Agrawal, Nonlinear Fiber Optics, Academic Press, 2001.

[11] R. Ryf, et al., "Mode-Division Multiplexing Over 96 km of Few-Mode Fiber Using Coherent $6 \times 6$ MIMO Processing,” J. Lightw. Technol, vol. 30, no. 4, pp. 521-531, 2012. 